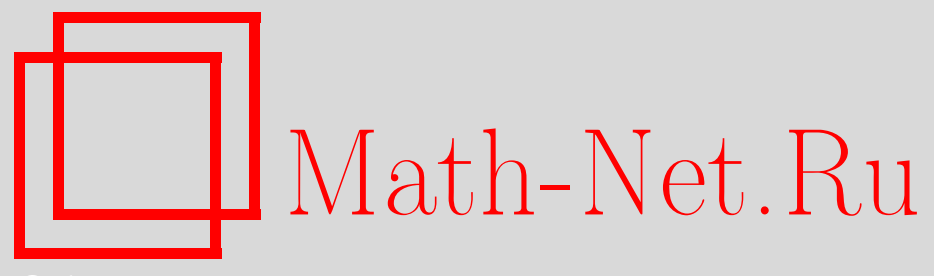

Ж. И. Абдуллаев, Конечность дискретного спектра при нетривиальных значениях полного квазиимпульса в системе трех бозонов на решетке, УМН, 2007, том 62, выпуск 1, 193-194

DOI: https://doi.org/10.4213/rm4468

Использование Общероссийского математического портала Math-Net.Ru подразумевает, что вы прочитали и согласны с пользовательским соглашением http://www . mathnet.ru/rus/agreement

Параметры загрузки:

IP: 54.92 .164 .108

26 апреля 2023 г., 11:03:35

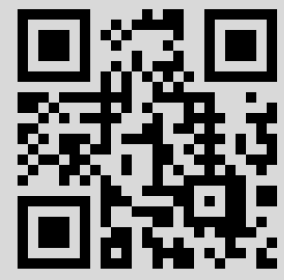




\section{Конечность дискретного спектра при нетривиальных значениях полного квазиимпульса в системе трех бозонов на решетке}

\section{Ж. И. Абдуллаев}

Конечность дискретного спектра непрерывного оператора Шрёдингера $H$ системы трех попарно взаимодействующих частиц при $\chi<0$ установлена в работах [1], [2]. Изучению случая $\chi=0$ посвящена работа [3], где было установлено, что даже при финитных взаимодействиях между частицами дискретный спектр оператора $H$ может быть бесконечным. При этом вопрос о бесконечности дискретного спектра оператора $H$ был связан с наличием так называемых виртуальных уровней в начале непрерывных спектров операторов $h_{\alpha}(\alpha=1,2,3)$, описывающих различные подсистемы из двух частиц. В этом случае [4] получена следующая асимптотика:

$$
\lim _{z \uparrow 0} \frac{N(z)}{|\ln | z||}=\mathscr{U}_{0} \quad\left(0<\mathscr{U}_{0}<\infty\right)
$$

где $N(z)$ - число собственных значений оператора $H$, лежащих ниже точки $z<0$, $\mathscr{U} 0$ зависит только от отношения масс частиц. В [5] доказана конечность дискретного спектра оператора $H$ при наличии виртуального уровня только у одного оператора $h_{\alpha}$.

Изучению аналогичных вопросов на решетке посвящена работа [6]. Непосредственное развитие работа [6] получила в [7]. Там доказано, что если двухчастичные операторы $h_{\alpha}(0) \equiv h(0), \alpha=1,2,3\left(h_{\alpha}(k)\right.$ - одинаковые, так как рассматриваются одинаковые частицы - бозоны) имеют виртуальные уровни в нуле, то у трехчастичного дискретного оператора Шрёдингера $H(0)$ имеется бесконечное число отрицательных собственных значений, накапливающихся к нулю. В этом случае существует выколотая окрестность $U_{\delta}^{o}(0)=\left\{p \in \mathbb{T}^{3}: 0<|p|<\delta\right\} \quad(\mathbb{T} \equiv(-\pi, \pi])$ такая, что при всех $K \in U_{\delta}^{o}(0)$ оператор $H(K)$ имеет лишь конечное число собственных значений, лежащих вне существенного спектра. Кроме того, получены аналогичные (1) асимптотики $N(0, z) \sim \mathscr{U}_{0}|\ln | z||$ при $z \rightarrow 0$, и $N(K, 0) \sim 2 \mathscr{U}_{0}|\ln | K||$ при $K \rightarrow 0$, где $N(K, z)$ - число собственных значений оператора $H(K)$, лежащих левее точки $z<\tau(K) \equiv \inf \sigma_{\text {ess }}(H(K))$.

В настоящей заметке дано доказательство конечности дискретного спектра оператора $H(K)$ для любого $K \neq 0$ при наличии виртуального уровня у оператора $h(0) \equiv$ $h_{\alpha}(0), \alpha=1,2,3$.

В импульсном представлении оператор энергии системы трех бозонов с парными контактными потенциалами притяжения действует в гильбертовом пространстве $L_{2}^{s}\left(\left(\mathbb{T}^{3}\right)^{3}\right)$ по формуле

$$
\begin{aligned}
& (H f)\left(k_{1}, k_{2}, k_{3}\right)=\left(\varepsilon\left(k_{1}\right)+\varepsilon\left(k_{2}\right)+\varepsilon\left(k_{3}\right)\right) f\left(k_{1}, k_{2}, k_{3}\right) \\
& \quad-\mu(2 \pi)^{-3} \sum_{\alpha=1}^{3} \int_{\left(\mathbb{T}^{3}\right)^{3}} \delta\left(k_{\alpha}-k_{\alpha}^{\prime}\right) \delta\left(k_{\beta}+k_{\gamma}-k_{\beta}^{\prime}-k_{\gamma}^{\prime}\right) f\left(k_{1}^{\prime}, k_{2}^{\prime}, k_{3}^{\prime}\right) d k_{1}^{\prime} d k_{2}^{\prime} d k_{3}^{\prime} .
\end{aligned}
$$

Здесь $\{\alpha, \beta, \gamma\}=\{1,2,3\}, \varepsilon(q)=\sum_{i=1}^{3}\left(1-\cos q_{i}\right)$ - энергия отдельного бозона с импульсом $q \in \mathbb{T}^{3}, \mu>0$ - энергия взаимодействия двух бозонов, $\delta(k)$ - трехмерная дельта-функция Дирака.

Оператор $H$ разлагается в прямой интеграл семейства самосопряженных операторов $\left\{H(K), K \in \mathbb{T}^{3}\right\}$. Оператор $H(K)$ называется оператором Шрёдингера и действует в гильбертовом пространстве $L_{2}^{s K}\left(\left(\mathbb{T}^{3}\right)^{2}\right)=\left\{f \in L_{2}\left(\left(\mathbb{T}^{3}\right)^{2}\right): f(p, q)=f(q, p)=\right.$ $f(p, K-p-q)\}$ по формуле (см. [6])

$$
(H(K) f)(p, q)=\varepsilon_{K}(p, q) f(p, q)-\mu(2 \pi)^{-3} \int_{\mathbb{T}^{3}}[f(K-p-q, s)+f(p, s)+f(s, q)] d s .
$$

Здесь $\varepsilon_{K}(p, q)=\varepsilon(K-p-q)+\varepsilon(p)+\varepsilon(q)$.

Работа выполнена при поддержке ФПФИ АН РУз (грант №67-04). 
Условия наличия виртуального уровня у оператора $h(0)$ равносильно условию $\mu=$ $\mu_{0}$, где $\mu_{0}=2(2 \pi)^{3}\left(\int_{\mathbb{T}^{3}}(\varepsilon(q))^{-1} d q\right)^{-1}$.

Теорема. Пусть $\mu=\mu_{0}$. Тогда для любого $K \neq 0$ оператор $H(K)$ имеет лишь конечное число собственных значений вне существенного спектра.

Как известно (см. [7]), существенный спектр оператора $H(K)$ состоит из отрезка $\left[\tau(K), \varepsilon_{\max }(K)\right]$, где $\varepsilon_{\max }(K)=\max _{p, q} \varepsilon_{K}(p, q)$ и для любого $K \neq 0$ имеет место неравенство $\tau(K)<\varepsilon_{\min }(K)$.

Пусть $T(K, z), z<\tau(K)$, - симметризованный оператор Фаддеева (см. [7]), соответствующий оператору $H(K)$, и он действует в $L_{2}\left(\mathbb{T}^{3}\right)$ по формуле

$$
(T(K, z) g)(p)=\frac{2 \mu_{0}(2 \pi)^{-3}}{\sqrt{\Delta(K-p, z-\varepsilon(p))}} \int_{\mathbb{T}^{3}} \frac{\left(\varepsilon_{K}(p, s)-z\right)^{-1} g(s) d s}{\sqrt{\Delta(K-s, z-\varepsilon(s))}},
$$

где $\Delta(k, z)$ - двухчастичный детерминант Фредгольма, который определяется по формуле $\Delta(k, z)=1-\mu_{0}(2 \pi)^{-3} \int_{\mathbb{T}^{3}}(\varepsilon(q)+\varepsilon(k-q)-z)^{-1} d q$.

Обозначим через $n(1, B)$ число собственных значений самосопряженного оператора $B$, лежащих правее точки 1 . Принцип Бирмана-Швингера $N(K, z)=n(1, T(K, z))$, $z<\tau(K)$ доказан в [7].

ДоказАтельство. Как известно, число $A=0$ является глобальным минимумом для регулярной функции $\Delta(K-p, \tau(K)-\varepsilon(p))$ на $\mathbb{T}^{3}$. Непосредственное вычисление показывает, что уравнение $\Delta(K-p, \tau(K)-\varepsilon(p))=0$ имеет конечное число решений в $\mathbb{T}^{3}$. Сначала рассмотрим случай, когда точки минимума функции $\Delta(K-p, \tau(K)-\varepsilon(p))$ являются невырожденными. В этом случае предельный оператор $T(K, \tau(K))$ является компактным и $T(K, z)$ сходится равномерно к $T(K, \tau(K))$ при $z \rightarrow \tau(K)$. Из неравенства Вейля $N(K, z)=n(1, T(K, z)) \leqslant n(1-\varepsilon, T(K, \tau(K)))+$ $n(\varepsilon, T(K, z)-T(K, \tau(K)))$ следует конечность числа собственных значений оператоpa $H(K)$, лежащих ниже существенного спектра (см. также [7]). Пусть минимумы функции $\Delta(K-p, \tau(K)-\varepsilon(p))$ являются вырожденными. Из неравенства Лоясеевича (см. [8]) следует, что изолированная экстремальная точка вещественно-аналитической функции является конечнократной, т.е. $\mid \Delta(K-p, \tau(K)-\varepsilon(p))-\Delta\left(K-p^{0}, \tau(K)-\right.$ $\left.\varepsilon\left(p^{0}\right)\right)|\geqslant C(K)| p-\left.p^{0}\right|^{m},\left|p-p^{0}\right|^{2}=\sum_{i=1}^{3}\left(p_{i}-p_{i}^{0}\right)^{2}$. А функция $\left(\varepsilon_{K}(p, q)-z\right)^{-1}$, участвующая в ядре интегрального оператора $T(K, z)$ принадлежит классу $C^{\infty}\left(\mathbb{T}^{3} \otimes \mathbb{T}^{3}\right)$ при всех $z \leqslant \tau(K)$. Поэтому $T(K, z)$ представляется в виде суммы двух самосопряженных операторов $T_{0}(K, z)$ и $T_{1}(K, z)$, в которой $T_{0}(K, z)$ - оператор конечного ранга $\ell$ при всех $z<\tau(K)$, а $T_{1}(K, z)$ компактно при $z=\tau(K)$ (см. [9]). Из неравенства Вейля имеем

$$
n(1, T(K, z)) \leqslant n\left(1-\varepsilon, T_{1}(K, z)\right)+n\left(\varepsilon, T_{0}(K, z)\right) .
$$

Первое слагаемое в правой части (2) конечно для любого $z<\tau(K)$ из-за компактности предельного оператора $T_{1}(K, \tau(K))$, второе слагаемое не превышает ранга $\ell$ оператора $T_{0}(K, z)$. Отсюда и из принципа Бирмана-Швингера $N(K, z)=n(1, T(K, z))$ следует утверждение теоремы.

Автор выражает благодарность проф. И. А. Икромову за обсуждению полученных результатов.

\section{Список литературы}

[1] Д. Р. Яфаев, Докл. АН СССР, 206:1 (1972), 68-70. [2] Г. М. Жислин, Докл. АН СССР, 207:1 (1972), 25-28. [3] Д.Р. Яфаев, Матем. сб., 94(136):4(8) (1974), 567-593. [4] A. V. Sobolev, Comm. Math. Phys., 156:1 (1993), 101-126. [5] Д. Р. Яфаев, ТМФ, 25:2 (1975), 185-195. [6] Ж. И. Абдуллаев, С. Н. Лакаев, ТМФ, 111:1 (1997), 94-108. [7] Ж. И. Абдуллаев, С.Н. Лакаев, ТMФ, 136:2 (2003), 231-245. [8] Б. Мальгранж, Идеаль дифференцируемых функиий, Мир, М., 1968. [9] И. А. Икромов, Ф. Шарипов, Функи. анализ и его прил., 32:1 (1998), 63-65.

Ж. И. Абдуллаев (Zh. I. Abdullaev)

Самаркандский государственный университет им. Алишера Навои

E-mail: jabdullaev@mail.ru
Представлено Р. А. Минлосом Принято редколлегией 11.08.2006 